\title{
Validation of vessel-based registration for correction of brain shift
}

\author{
I. Reinertsen $^{\text {a,* }}$, M. Descoteaux ${ }^{\text {b,1 }}$, K. Siddiqi ${ }^{\text {b }}$, D.L. Collins ${ }^{\text {a }}$ \\ ${ }^{a}$ Montreal Neurological Institute (MNI), McGill University, Montréal, Canada \\ ${ }^{\mathrm{b}}$ Center for Intelligent Machines (CIM), McGill University, Montréal, Canada
}

Received 16 February 2006; received in revised form 11 April 2007; accepted 11 April 2007

Available online 19 April 2007

\begin{abstract}
The displacement and deformation of brain tissue is a major source of error in image-guided neurosurgery systems. We have designed and implemented a method to detect and correct brain shift using pre-operative MR images and intraoperative Doppler ultrasound data and present its validation with both real and simulated data. The algorithm uses segmented vessels from both modalities, and estimates the deformation using a modified version of the iterative closest point (ICP) algorithm. We use the least trimmed squares (LTS) to reduce the number of outliers in the point matching procedure. These points are used to drive a thin-plate spline transform to achieve non-linear registration. Validation was completed in two parts. First, the technique was tested and validated using realistic simulations where the results were compared to the known deformation. The registration technique recovered $75 \%$ of the deformation in the region of interest accounting for deformations as large as $20 \mathrm{~mm}$. Second, we performed a PVA-cryogel phantom study where both MR and ultrasound images of the phantom were obtained for three different deformations. The registration results based on MR data were used as a gold standard to evaluate the performance of the ultrasound based registration. On average, deformations of $7.5 \mathrm{~mm}$ magnitude were corrected to within $1.6 \mathrm{~mm}$ for the ultrasound based registration and $1.07 \mathrm{~mm}$ for the MR based registration.
\end{abstract}

(C) 2007 Elsevier B.V. All rights reserved.

Keywords: Brain shift; Doppler ultrasound; Registration

\section{Introduction and motivation}

\subsection{Neuro-navigation and brain shift}

Modern image guided neurosurgery (IGNS) systems enable the surgeon to navigate within the patient's brain using pre-operative anatomical images (MRI, CT) as a guide. The pre-operative images are related to the patient using a rigid body transformation calculated from a number of anatomical landmarks that can be easily identified on both the patient's head and the pre-operative images. By using a computer-tracked probe during the procedure,

\footnotetext{
${ }^{*}$ Corresponding author. Present address: SINTEF Health Research and National Center for 3D Ultrasound in Surgery, St. Olav University Hospital, Trondheim, Norway. Tel.: +1 47 90212159; fax: +1 47 93070800.

E-mail address: Ingerid.Reinertsen@sintef.no (I. Reinertsen).

${ }^{1}$ Present address: Odyssee Team, INRIA Sophia-Antipolis, France.
}

the surgeon can localize any point in the patient's brain on the pre-operative images. A significant source of error in these systems is brain tissue movement and deformation, so called brain shift, during the procedure. Tissue movement can be caused by gravity, drainage of cerebro-spinal fluid (CSF), retraction and resection of tissue, swelling of brain structures, and administration of drugs. The amount of movement and its influence on the accuracy of the neuro-navigation system depend on a number of factors including surgical target size and location, craniotomy size and patient position during surgery.

The magnitude and direction of brain deformation during surgery have been the subject of several studies. The first quantitative measurements of brain deformation during surgery relied on recordings of points on the cortical surface relative to fixed points on the cranial surface Hill et al., 1998; Roberts et al., 1998. These studies showed a cortical surface shift of $10 \mathrm{~mm}$ on average, and movement was found to be greatest along the direction of gravity. 
To better describe the dynamic process of brain deformation, several groups have used intraoperative MRI (iMRI) to study brain shift Nabavi et al., 2001; Hartkens et al., 2003. The results show that surface shift ranges from almost no detectable shift for smaller lesions to up to $50 \mathrm{~mm}$ for larger lesions. Surface shift well beyond the craniotomy has also been documented. As in the previously discussed studies it was found that surface shift was mainly due to loss of cerebro-spinal fluid and resulted in a shift in the direction of gravity. They also showed that surface shift occurs throughout the procedure while deformation of deeper structures occurs mainly during resection. Volume changes depend on the nature of the surgical procedure, and are in general greater for resection cases than for biopsies and functional interventions. The principal direction of displacement was not always aligned with the direction of gravity.

Intraoperative ultrasound has also been used to estimate brain shift. Letteboer et al. (2005) used ultrasound to measure the linear component of the shift at the tumor boundary. This study also confirms the assumption that the brain deforms mainly in the direction of gravity is not always valid.

In summary, the cortical surface shift is mainly caused by loss of CSF and subsequent "sinking" of the brain in the direction of gravity. Surface shift can occur well beyond the borders of the dural opening and can occur throughout the procedure. However, the surface has been shown to settle in cases where the resection cavity is smaller than the cortical opening. If the cavity is larger than the cortical opening, the borders sink in to form a crater. Deformation of subsurface structures on the other hand is mainly due to resection, relief of weight and intraparenchymal pressures. Larger deformations are generally observed in the hemisphere ipsi-lateral to the lesion, but significant deformations can also occur in the contra-lateral hemisphere.

In the following sections, we present a detailed overview of previous work applied to the detection and correction of brain shift. We present our vessel based registration technique in Section 2 followed by a series of validation experiments in Section 3.

\subsection{Model based techniques}

With all this prior knowledge about how the brain shifts and deforms during surgery, a number of groups have developed model-based techniques to try to correct for the displacements. Among the first groups to attempt this approach was Miga et al., 1999. The technique was applied to four neurosurgical cases, and it was found that the model could account for $79 \%$ of the gravity induced deformations on average. Other groups have extended this work to include more complex deformations and deformation of deeper structures Roberts et al., 1998; Skrinjar et al., 2002. In these studies, it was assumed that brain tissues are isotropic, homogeneous and with identical density and stiffness. It was also assumed that there is no deformation in the hemisphere contralateral to the craniotomy, and that all deformations can be estimated based on data from the exposed surface.

In general, the displacement and deformation of the brain during surgery is far more complex and far reaching than these models assume, and more work is needed to estimate the mechanical properties of the brain Soza et al., 2004 in order for this type of approach to be useful in more than a very limited number of neurosurgical procedures.

The more direct solution to the problem is to acquire new images when significant amount of deformation is suspected. The most popular intraoperative imaging modalities for neurosurgery are intraoperative CT, intraoperative MRI, and intraoperative ultrasound (US) imaging.

\subsection{Intraoperative $C T$ imaging}

A few groups have used intraoperative CT to actualize the navigation data and verify the anatomical situation during surgery Haberland et al., 2000; Grunert et al., 1998. The CT images can be used to localize intracranial lesions, but suffer from lower soft tissue contrast than MRI, and are therefore less useful for brain surgery. CT imaging is more commonly used in spine surgery, where the vertebrae and surrounding structures are of primary interest. Other disadvantages of intraoperative $\mathrm{CT}$ imaging are the radiation dose to the patient which limits the number and duration of the scans, and the physical space occupied by the scanner in the operating room.

\subsection{Intraoperative $M R$ imaging}

Intraoperative MRI (iMRI) scanners can provide the surgeon with updated anatomical images several times during a procedure, and can therefore be a valuable tool for characterization and correction of brain shift. One of the first reports on the use of iMRI for neurosurgical guidance was presented by Black et al. (1999). They illustrated the advantages of intraoperative MRI imaging in a series of 60 craniotomies for tumor resection. Images were acquired before and after opening of the dura and after closure of the craniotomy. Nimsky et al. (2001) went one step further and used intraoperative data for registration purposes. Intraoperative MR images were rigidly registered to the pre-operative data using MR-visible fiducials placed around the craniotomy. The root mean square position error after registration was reported to be between $0.39 \mathrm{~mm}$ and $2.3 \mathrm{~mm}$.

An image based registration algorithm for iMRI was presented by Ferrant et al. (2002). A biomechanical finite element (FE) model driven by surface correspondences was used to estimate the deformation of the entire brain during surgery. The accuracy of the registration was evaluated using manually identified landmarks and resulted in a mean error of less than $1.6 \mathrm{~mm}$. A second image based registration technique was published by Hastreiter et al. (2004). After having characterized the brain deformations, they 
used a non-linear registration method based on mutual information to register pre-operative and intra-operative data. The registration process made it possible to register pre-operative functional data such as fMRI, PET and MEG to the intraoperative MR images in 20-30 min.

Even though intra-operative MR imaging provides good quality images in reasonable time, this solution suffers from a number of disadvantages Nimsky et al., 2004; Jolesz, 2005. Intra-operative MR imaging is a complex, expensive and sometimes quite a time consuming procedure. The intraoperative images may be of poorer quality than pre-operative MR images due to scanner design and short acquisition time. In general, intraoperative images are less complete, have lower resolution and are more susceptible to image distortions due to inhomogeneous magnetic fields when compared to pre-operative images. Another major shortcoming of this solution is the substantial financial investment required for the scanner as well as MR-compatible surgical instruments. These investments are justifiable for only a very limited number of hospitals. In addition, interventional MR scanners are space-consuming and in many cases compromise the surgeon's access to the operating field.

\subsection{Intraoperative ultrasound imaging}

Intraoperative ultrasound imaging does not suffer from many of the limitations associated with interventional MRI. A high-end ultrasound scanner costs less than $10 \%$ of a typical MRI system and is already in use by many neurosurgeons. In addition, ultrasound systems are portable and compatible with existing surgical equipment. Despite these advantages, the use of ultrasound in neuro-navigation has been limited, probably due to poor image quality and the difficulty of interpreting such images.

Since the mid-1990s a number of groups have developed systems correlating intraoperative US with pre-operative MR. In a neurosurgical context, intraoperative ultrasound imaging can either be used directly as a surgical guide when brain shift occurs or as a registration target for the preoperative images in order to correct for deformations. These systems are described in more detail in the following background sections before presenting our registration method and validation experiments.

\subsubsection{Direct ultrasound navigation}

Grønningsæter et al. (2000) developed a neuro-navigation system based on navigation solely by $3 \mathrm{D}$ ultrasound. This system also incorporates visualization of pre-operative MR and/or CT images, but uses only intra-operative 3D ultrasound for navigation if brain deformation occurs. Navigation by ultrasound images requires high quality images and display software in addition to well trained surgeons and technicians.

\subsubsection{Manual registration of intraoperative ultrasound}

Intra-operative ultrasound data can also be used in a less direct manner. Image registration techniques can be used to update pre-operative data. By registering pre-operative MR or CT images with intra-operative ultrasound images, complex deformations can be estimated and accounted for in the navigation system. For example, by identifying anatomical landmarks in the US images, and using a physical model of the brain, an elastic transformation can be calculated and applied to the pre-operative data. Comeau et al. (2000) presented a surgical guidance system that incorporated pre-operative images with intraoperative ultrasound to detect and correct for brain shift during neurosurgical procedures. Two dimensional ultrasound images were acquired during the operation and compared to the corresponding slice from the pre-operative data set. A method was presented to manually identify homologous landmarks in ultrasound and MRI in order to construct a set of displacement vectors that would allow the pre-operative MR image to be warped to match the intra-operative ultrasound image. The mapping procedure was demonstrated to have an accuracy better than $2 \mathrm{~mm}$. Gobbi et al. (2000) demonstrated a similar technique where manually placed landmarks and a thin-plate spline interpolation were used to deform the MR volume to match the ultrasound volume.

\subsubsection{Automatic registration of intraoperative ultrasound}

Several automatic registration procedures have also been developed, in order to minimize the need for user intervention and speed up the procedure, which is particularly important for intraoperative registration. Roche et al. (2001) estimated the rigid body transform required to linearly align pre-operative MR images and intra-operative US images. They correlated the US intensities with both the MR intensity and the MR gradient magnitude using a variant of the correlation ratio and a robust distance measure. The algorithm was tested on two clinical datasets and one phantom dataset. Because no gold standard was available, registration loops involving both the ultrasound and MR data were used. In the ideal case each loop should lead to the identity matrix. They reported registration residuals up to $1.65 \mathrm{~mm}$ in translation and $1.57^{\circ}$ in rotation and a computation time of 5-10 min.

In order to correct for non-linear deformation Arbel et al., 2001; Arbel et al., 2001 used a tracking system to reconstruct $3 \mathrm{D}$ volumes from a series of US images in the same space as the pre-operative MR-image. From the pre-operative MR images, they created pseudo-US images that closely resembled real US images of the same structures acquired during surgery. They then used an intensity based non-linear registration technique to match tracked intraoperative US images with the pseudo-US images to detect and correct brain deformations. Qualitative results from 12 surgical cases showed that the technique was able to account for a large portion of the deformations.

Registration of intraoperative US with pre-operative MR is a challenging registration problem due to very different underlying physical principles and thus different image characteristics. Image intensities, noise characteristics, con- 
trast, volume coverage and dimensionality are only a few main differences between a typical pre-operative MR image and a corresponding intraoperative ultrasound acquisition.

\subsection{Vessel based registration}

To try to overcome some of the difficulties discussed in the previous section, we explore a different approach to this particular registration problem. The idea is to use homologous features in the two datasets as "landmarks". Such features might be any segmented structures present in both images such as organ surfaces and vascular structures. In this project we investigate the use of blood vessels segmented from pre-operative angiographic images and Doppler US for registration purposes. The cerebral vasculature is relatively easy to identify and segment from preoperative angiographic data such as MR angiograms (MRA). A method to segment vessels from other types of MR acquisitions such as proton density (PD) images or gadolinium $(\mathrm{Gd})$ enhanced MR images has been presented in Descoteaux (2004). Segmentation of Doppler ultrasound images can easily be performed by simple thresholding although this often produce vessels with a too big radius due to noise from moving vessel walls. By using the centerlines of the vessels this problem is largely overcome.

The cerebral vasculature is a good candidate for use in image registration because the vessels are distributed all over the cerebral cortex and inside the brain and move with the surrounding tissue. The brain deformations are therefore well captured by the vasculature. In addition, blood vessels will be present in any region of interest (ROI) throughout the brain. The probability of not finding reliable landmarks in a given ROI is therefore low. Keeping track of important vessels during surgery also provides the surgeon with important reference points in order to avoid major vessels during the procedure and monitor blood supply to specific areas of the brain. This approach has already been investigated by a number of different groups for several different purposes. Porter et al. (2001) rigidly registered MRI with B-mode and color Doppler ultrasound volumes based on segmented blood vessels from the forearm, the liver and a prostate phantom. The skin surface, bone and internal landmarks were used to evaluate the registration error which ranged from 2 to $8 \mathrm{~mm}$.

Another rigid body registration technique based on vasculature was presented by Slomka et al. (2001). The carotid bifurcation of six patients was imaged with B-mode and Power Doppler ultrasound as well as MRA. The mean errors were $0.32 \mathrm{~mm}$ in translation and $1.6^{\circ}$ in rotation based on a series of anatomical landmarks for initial misalignments of up to $5.4 \mathrm{~mm}$ in the $x$ and $y$ directions, $10 \mathrm{~mm}$ in the $z$ direction and rotations up to $40^{\circ}$. The algorithm was not affected by missing arterial segments of up to $8 \mathrm{~mm}$, but would fail if the bifurcation was missing from either dataset.

A third rigid body registration technique as well as a vessel segmentation algorithm was presented by Aylward et al. (2003). A registration metric was defined based on the parameters of the vessel segmentation algorithm and used to register $\mathrm{CT}$ images of the liver and pre and postsurgery MRA images of the brain. A series of Monte Carlo simulations was conducted to measure how consistently the registration method was able to align segmented vessels from the liver given random initial misregistrations. The application of this registration algorithm was extended to include CT to ultrasound registration Aylward et al., 2002 and then further extended to take into account nonlinear deformations Jomier and Aylward, 2004. Following global rigid registration, each branch in the vessel tree was linearly registered resulting in a piece-wise rigid transformation. The alignment was then further refined with a deformable registration method. The results showed that the $87 \%$ of the centerline points in the model were within two voxels of the centerlines in the target image.

A more recent technique to register $\mathrm{MR}$ and $\mathrm{B}$-mode ultrasound images of the liver based on vasculature was presented by Penney et al. (2004). The rigid registration used ultrasound images to establish the correspondence between the MR volume and the patient on the operating table. This corresponds to the rigid registration usually performed by identifying homologous landmarks on the patients head and on the pre-operative images before neurosurgical procedures. The results showed that the method was accurate to within an RMS error of between 2.3 and $5.5 \mathrm{~mm}$ with respect to a "bronze standard" registration calculated by manually picking points in both modalities.

The algorithm described in this paper is designed to register pre-operative MR images and intra-operative US images of the brain in order to correct the brain shift occurring during neurosurgical procedures. The work is based on experiments first presented in Reinertsen et al. (2004) where we demonstrated that it was possible to use vesselbased non-linear registration for this task. In this paper, we have further developed and improved our vessel based registration method and present experimental validation of the technique. We have replaced the free-form ANIMAL-based deformation Collins and Evans, 1997 with a thin-plate spline transform to improve regularization of the deformation. We now use a modified version of the ICP algorithm to register vessel centerlines extracted from MR and Doppler ultrasound data. In order to reduce the number of outliers, we have incorporated the least trimmed squares (LTS) robust estimator Rousseeuw and Leroy, 1987. Therefore, our method effectively reduces the number of incorrect pairings without limiting the capture range of the registration algorithm. While our algorithm shares some similarities with the procedure described by Lange et al. (2004), there are some important differences. Our procedure is applied to interventional brain imaging, while Lange's technique was applied to liver. Both techniques use segmented vessel centerlines to drive the registration. Our technique uses LTS robust estimation to reject outlier points instead of a the user-defined distance threshold used by Lange. Both techniques use spline-based regularization 
of the deformation; we use a thin-plate spline while Lange uses B-splines. Finally, Lange estimated the quality of registration quantitatively based on the RMS distance of vessel-points and semi-quantitatively based on structure boundaries. Our main contribution in this paper is a more thorough quantitative validation using data with simulated deformations and real MR and US data from a novel deformable anthropomorphic poly vinyl alcohol cyrogel (PVAc) brain phantom.

This paper is organized into five sections. In Section 2, the vessel segmentation method and the centerline extraction technique are briefly described, and the registration algorithm is presented in detail. Section 3 is concerned with the validation experiments using simulated and phantom data. A discussion of the results is given in Section 4, and finally our conclusions are presented in Section 5.

\section{Methods}

\subsection{MR vessel segmentation}

We used a new multi-scale geometric flow for segmenting vasculature in the MR images of the phantom. The method can be summarized in three steps: First, the method applies Frangi's vesselness measure Frangi et al., 1998 to find putative centerlines of tubular structures along with their estimated radii and orientation. Second, this multi-scale measure is distributed to create a vector field which is orthogonal to vessel boundaries. Finally, the flux maximizing flow algorithm Vasilevskiy and Siddiqi, 2002 is applied to the vector field to recover the vessel boundaries. This technique overcomes many limitations of existing approaches in the literature specifically designed for angiographic data due its multi-scale tubular structure model. It has a formal motivation, is topologically adaptive due to its implementation using level set methods, is computationally efficient and requires minimal user interaction. The technique is detailed in Descoteaux (2004).

\subsection{US vessel segmentation and volume reconstruction}

When scanning using Doppler ultrasound imaging, the Doppler signal and the B-mode signals are combined on the display of the ultrasound scanner. The Doppler signal is displayed in color, and the B-mode signal is displayed in grayscale. Segmentation of the ultrasound images was therefore obtained by extracting all colored pixels from the original images. A simple filter was implemented that would set to zero all pixels with a saturation equal to zero (Hue-Saturation-Value color model), which constitutes the grayscale. Following segmentation, the 2D images were masked to remove information outside the ultrasound wedge, converted to grayscale, and finally reconstructed into a 3D volume. The 3D volume was then thresholded again to produce a binary image. An example of an ultrasound image before and after segmentation and after masking is shown in Fig. 1. The 2D slices were interpolated to a uniform grid using a Kaiser-Bessel function as the interpolation function and an isotropic regrid radius of $2 \mathrm{~mm}$.

\subsection{Centerline extraction}

Following segmentation, we extracted the vessel centerlines using a fast, robust and automatic method based on medial surfaces. The technique uses the average outward flux of the gradient vector field of the distance transform of the object to compute the medial surface Siddiqi et al., 2002. The centered medial curves are then obtained by topology preserving thinning ordered by the distance function to the object's boundary. This ensures that the remaining points lie on the medial surface and as far away from the vessel boundary as possible. The medial curve was finally pruned based on length to remove superfluous branches and obtain a single curve for each vessel branch. Details on the method can be found in Bouix et al. (2005).

\subsection{Registration algorithm}

After segmentation and centerline extraction, the MRA image and the Doppler ultrasound volumes are binary images representing the vascular tree. The vessels are in the form of a "skeleton" representing the midlines. The two datasets are only partially overlapping, and vessels are not necessarily continuous. A number of vessels might also be missing from one or both data sets. The task of registering the two datasets using a modified version of the
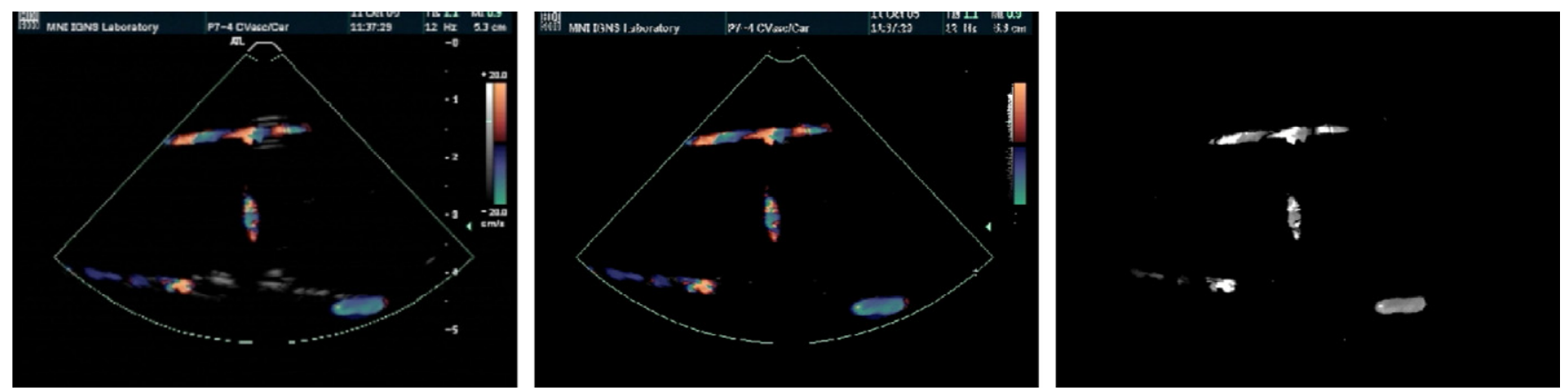

Fig. 1. An example of an ultrasound image before (left) and after segmentation (middle) and after masking (right). The masking removes the text and the colorbar, and keeps only the trapezoid-shaped ultrasound image. 
original ICP algorithm presented by Besl and McKay (1992), can be summarized in the six following steps as proposed by Rusinkiewicz and Levoy (2001):

(1) Sampling.

(2) Point matching.

(3) Weighting/rejecting point pairs.

(4) Estimating the transformation.

(5) Applying transformation to the source points.

(6) Calculating the error.

\subsubsection{Sampling}

The original ICP algorithm attempts to use all available points to establish correspondence when computing the transformation. In order to decrease computational cost, improve the convergence rate, and reduce sensitivity to noise and missing data, some authors have proposed to use a subset of all available points. There are a number of sampling strategies in the literature that have been presented in order to adapt to particular types of images. Turk and Levoy (1994) created triangle meshes from laser range images and used the ICP algorithm to bring corresponding portions of meshes from different images into alignment with one another in order to create a single polygonal mesh that completely describes the outside part of the scanned object. The creation of triangle meshes represents a uniform sub-sampling of the images. Sub-sampling of the images is an efficient way of reducing computation time, but depending on the sampling frequency, accuracy may be compromised. A different method of sub-sampling is to choose a number of points extracted at random. Masuda et al. (1996) used this technique with a different subset of points at each iteration in order to provide different starting positions for the algorithm. Other possibilities include selecting points with high intensity gradients Weik, 1997 or choosing points such that the distribution of normals among selected points is as large as possible Rusinkiewicz and Levoy, 2001. in order to obtain more points in regions with small features critical to determining the correct alignment. In line-to-line registration, such features could be regions with high curvature or bifurcations. In this paper we start with all available source points and then selects points based on the distance to the target. As an option it is possible to perform a uniform sub-sampling of the selected points in order to speed up the computation.

\subsubsection{Point matching}

The next step addresses the problem of finding corresponding points in the source and target point sets. Because the ICP algorithm is sensitive to the source vs. target selection, the source should always have fewer points than the target. In the original ICP algorithm, the simple Euclidean distance was used to find the closest point in the target dataset. The closest-point algorithm tends to produce a large number of incorrect pairings when the images are relatively noisy or do not completely overlap. This sensitivity to noise and missing data is one of the main disadvantages of the original ICP algorithm. Because noise and missing data are problems frequently encountered in real images, a number of point matching techniques have been developed in order to increase the robustness of the registration algorithm. Possible approaches used in the past are to find the intersection of the ray originating at the source point in the direction of the source point's normal with the destination surface, or different projection methods such as projection of the source point onto the target followed by a local search based on distance or intensity Pulli, 1999. Rusinkiewicz and Levoy (2001) found that the projectionbased algorithms converged significantly faster than for example the closest point method. In their experiments, convergence was reached in between 10 and 20 iterations. In the experiments presented in this paper, we reached convergence in less than 35 iterations in all cases, and linear registration was completed in less than $15 \mathrm{~s}$ which was considered satisfactory. We have therefore chosen to keep the original point matching technique based on the Euclidean distance and minimize the number of incorrect pairings by implementing the least-trimmed squares estimator as explained in the following section.

\subsubsection{Weighting and rejecting point pairs}

The idea behind the assignment of weights or completely discarding certain point pairs is to limit as much as possible the influence of erroneous pairings on the transform computation. Efforts are made to reduce the number of such pairs through sampling and point matching strategies, but when dealing with noisy data where the overlap is not complete and data are missing as is the case here, efficient weighting and rejection techniques may considerably improve the final result. Without any weighting and/or rejection strategy, all pairs will be used and all points will be equally weighted. A simple modification to this method is to assign lower weights to pairs with greater point-topoint distance, and to possibly reject corresponding points more than a given distance apart. Another method proposed in the past is weighting based on the compatibility of normals. The weight is then calculated as the scalar product of the normals. Point pairs with colinear normals will have weights equal to one, and point pairs with perpendicular normals will be rejected.

Other strategies include rejection of pairs whose pointto-point distance is larger than some multiple of the standard deviation of distances, or rejection of pairs that are not consistent with neighboring pairs. A potentially very useful strategy is to remove pairs that include points on boundaries. These pairs may introduce a systematic bias in the estimated transform in cases where the overlap is not complete.

A method widely used in computer vision is the random sample consensus (RANSAC) algorithm introduced by Fischler and Bolles (1981). The method selects a subset of the data to estimate the parameters of the model to fit. The subset is selected at random, and the algorithm determines the number of samples that are within an error 
tolerance. If the number of samples within the error tolerance is high enough, the solution is kept. The process is repeated and the solution with the smallest error is kept as the final model. The number of iterations required increases with the size of the sample subset and the percentage of outliers in the data.

Another possibility is to use robust regression methods such as the least median of squares (LMS) Trucco et al., 1999 or the least trimmed squares (LTS) Chetverikov et al., 2002. While the least squares technique minimizes the sum of squared residuals, the LMS minimizes the median of squared residuals. The LTS method on the other hand, is based on sorting and trimming the sequence of squared residuals. The squared residuals are sorted, and the points corresponding to the $n \%$ greatest distances are rejected. The percentage is user defined and can be adjusted according to the amount of noise or missing data expected in the dataset. The transformation is then calculated based on the remaining pairs, and the result is applied to the entire dataset. These two steps are then iterated until convergence. The LTS method is usually preferred to the LMS because it has a better convergence rate and a smoother objective function Rousseeuw and Leroy, 1987. LTS and LMS have the same breakdown point of $50 \%$, which means that the number of outliers in the dataset cannot exceed $50 \%$.

\subsubsection{Transformation}

Most of the registration methods using a variant of the ICP algorithm estimate a rigid body transform (three translations and three rotations). For linear registration, we have also included isotropic scaling, which gives a total of seven parameters. While this might be sufficient in cases of motion detection or to provide a good starting position for non-linear registration, it is not enough to describe the highly complex brain deformation taking place during neurosurgical interventions. The deformation is non-linear, and single points can move as far as $50 \mathrm{~mm}$ from their initial position. One possibility is to use a thin-plate spline (TPS) transformation Bookstein, 1989. TPS is an interpolation method that finds a "minimally bended" smooth (hyper)surface that passes through all given points. TPS are particularly popular in representing shape transformations, for example in image morphing or shape detection. In this work, we use the thin-plate spline transformation with points selected as described above, to represent the non-linear component of the deformations. The interpolation can be regularized using a scaling parameter $\sigma$ that will determine the "stiffness" of the spline. In this work, we start by estimating a seven parameter linear registration. In neuronavigation, this linear transformation is required due to the error in the landmark based registration performed prior to the opening of the skull and the actual linear component of the brain deformation occurring after the craniotomy. Then, the linear registration is refined by rerunning the algorithm and using a thin-plate spline transform to correct the non-linear component of the deformation.

\subsubsection{Registration error}

In the original ICP algorithm, the mean squared error was used and the algorithm was proved to converge to a local minimum of the objective function in terms of this error metric. A "point-to-plane" metric can also be used by taking the sum of squared distances from each source point to the plane containing the target point and oriented perpendicular to the target normal Chen and Medioni, 1991. The robust estimators LMS and LTS also converge to a local minimum of the objective function depending on the starting position Rousseeuw and Leroy, 1987. The thin-plate spline transform needs to have a reasonably good starting point, in terms of correct point pairings in order to give a satisfactory result. In most cases involving registration of pre-operative MR and intra-operative ultrasound, the two modalities will be linearly registered at the beginning of the procedure. This initial registration can be corrected by performing a linear ICP registration with seven parameters as described above. In cases where there is no initial linear registration, or if the ultrasound probe is not tracked during the procedure, it is possible to manually perform a coarse linear registration by dragging the source dataset into place.

For the linear registration, the steps described in Section 2.4.1, 2.4.2, 2.4.3, 2.4.4, 2.4.5 need to be iterated until a stopping criterion has been met. In previous literature, this criterion is usually a fixed number of iterations, an error metric below a pre-defined threshold or the difference between two successive error measurements below a predefined threshold. In this work, the iterations are stopped when the difference in mean distance between the source points and their closest target point between two successive iterations is smaller than $0.1 \mu \mathrm{m}$. Possible limitations with this approach are that the difference in distance might not fall below this limit, and that the optimizer could oscillate between two local minima. In the experiences presented in this paper, however, the algorithm has converged to a correct solution in all cases.

\subsubsection{Algorithm}

In this project, we have chosen to use the least trimmed squares and the simple Euclidean distance for point matching. The algorithm can be summarized in four steps:

(1) Find the closest point in the target dataset for each source point.

(2) Sort the distances, and select the source points corresponding to the $n \%$ smallest distances.

(3) Estimate a seven parameter linear transform or a thin-plate splines deformation based on the selected points.

(4) Apply the transformation to the entire dataset.

\section{Experiments and results}

In order to validate the registration algorithm presented above we performed two sets of experiments. First, we sim- 
ulated 14 realistic brain deformations in order to test the algorithm in a situation where the ground truth is known. Second, we performed a phantom study with a deformable brain phantom in order to come closer to a realistic clinical situation and to test the registration technique with real ultrasound data.

\subsection{Simulations}

\subsubsection{Data pre-processing}

For the simulation experiments, a standard phase-contrast MRA $\left(\mathrm{TE}=71 \mathrm{~ms}, \mathrm{TR}=8.2 \mathrm{~ms}\right.$, flip angle $\left.=15^{\circ}\right)$ from a normal volunteer with full brain coverage and a voxel size of $0.5 \times 0.5 \times 1.5 \mathrm{~mm}$ was used. The original dataset was resampled using tri-linear interpolation to an isotropic voxel size of $0.5 \mathrm{~mm}$. A series of 22 landmarks were placed at random spots throughout the volume and four landmarks were placed on the surface of the cortex in a square just above and below the right lateral fissure. These four landmarks were then manually displaced from \pm 2 to $20 \mathrm{~mm}$ in the $x$-direction (left-right direction). These deformations represent a smooth expansion or contraction toward the midline of the brain as shown in Fig. 2. A thinplate spline transform was computed between the original 26 landmarks and the 26 landmarks where four points had been displaced. The resulting transform was then applied to the resampled MRA dataset in order to obtain a deformed version of the same brain. Thus, the thin plate spline transform represents the ground truth in these experiments, and the two datasets (resampled and deformed) will be used to estimate the deformation.

In order to simulate a typical ultrasound acquisition, a $4.5 \mathrm{~cm}$ rectilinear scan path was defined between two points on the cortical surface in the region of interest on the MRA. The region of interest is shown by the rectangular box in Fig. 2. Two points inside the brain were manually selected to determine the direction of the first and last image plane. The orientation of the image planes was perpendicular to the scan path. The dimension of the image planes was $50 \mathrm{~mm}$ wide, a depth of $40 \mathrm{~mm}$ and a thickness of $3 \mathrm{~mm}$, with a voxelsize of $1 \times 1 \times 3 \mathrm{~mm}$ and $2 \mathrm{~mm}$ between each slice. The slice was averaged over $3 \mathrm{~mm}$ in the scan direction to simulate the thickness of the ultrasound beam. The individual slices were thresholded to
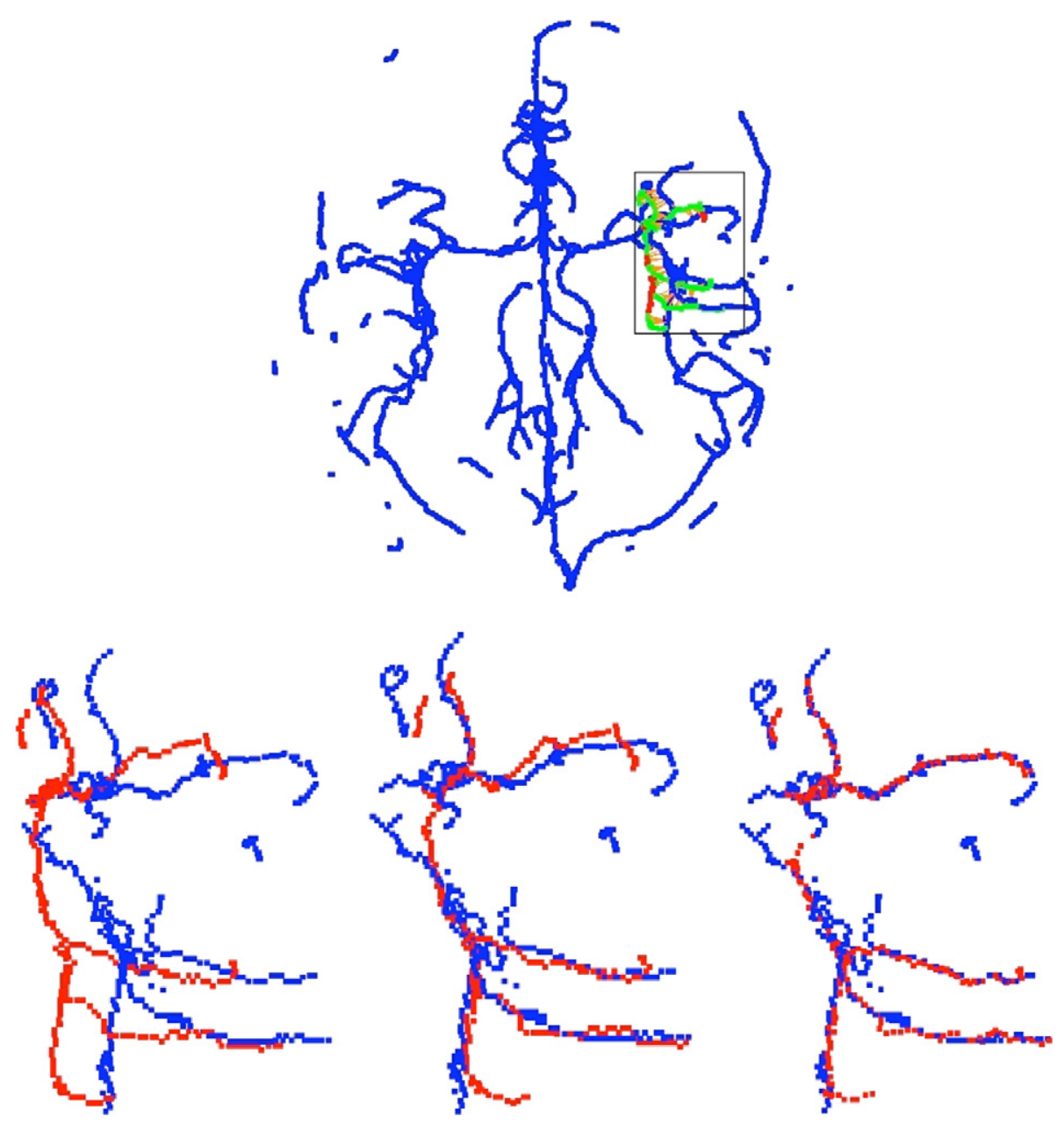

Fig. 2. The centerlines extracted from the simulated ultrasound volume registered to the MR vessel tree (top). Before registration (bottom left), after linear registration (bottom middle) and after non-linear registration (bottom right). In the upper image target points are in blue, source points that participate in the registration are in green and source points that do not participate in the registration are in red. The yellow lines illustrate the closest point correspondences for the points that participate in the registration. 
segment vessels and then masked using a wedge-shaped mask to simulate the shape of real ultrasound images. The masked images were then reconstructed into a volume using the reconstruction algorithm described in Section 2.2. Following volume reconstruction centerlines were extracted. The original MRA dataset was then segmented using the algorithm described in Section 2.1. The vessel centerlines were extracted from the segmented data, and used as input to the registration algorithm. In order to reduce the noise in the extracted centerlines (single points not connected to the vessel tree), points located more than $5 \mathrm{~mm}$ from their closest neighbor were removed from the image prior to registration. The resulting vessel tree is shown in blue ${ }^{3}$ in Fig. 2.

This technique of simulating ultrasound data from MR images is similar to the method proposed by Arbel et al. (2001). They created pseudo ultrasound data from preoperative MR images in order to facilitate intensity based registration of intra-operative ultrasound data.

\subsubsection{Registration}

In this experiment the simulated ultrasound volume represents a part of the middle cerebral artery that has been shifted and deformed compared to the target image which represents a nearly complete arterial "tree". Because the simulated ultrasound volume contained fewer points than the original MR dataset, it was considered the source image in this experiment. In order to recover the deformation, the source image was first linearly registered to the target in order to provide an optimal position for the non-linear deformation. In this step, between $80 \%$ and $99 \%$ of the available source points were used. The iterations were stopped when the difference in mean distance between all source points and their closest target point between two successive iterations was smaller than $0.1 \mu \mathrm{m}$. The mean distance as a function of iteration number for all 14 linear registrations is shown in Fig. 3.

The registration was then further refined by non-linear registration, where $55-99 \%$ of the source points were used and a $\sigma$ between 0.5 and 1.5. An example of the registration is shown in Fig. 2. To evaluate the performance of the registration technique, the recovered transformations were compared with the ground truth thin plate spline transform applied. We computed the 3D root-mean-square (RMS) of the difference between the two transforms over every third voxel in the region of interest (ROI). In addition, a series of 10 landmarks placed in the highly deformed region were used to specifically estimate registration accuracy in the ROI. The percentage of the deformation recovered by the registration algorithm was calculated in each case using the following formula:

$\%=\frac{\left(\mathrm{RMS}_{\text {Before }}-\mathrm{RMS}_{\text {After }}\right) \times 100}{\mathrm{RMS}_{\text {Before }}}$.

For the landmarks, the RMS in Eq. (1) should be replaced by the mean distance between landmarks.

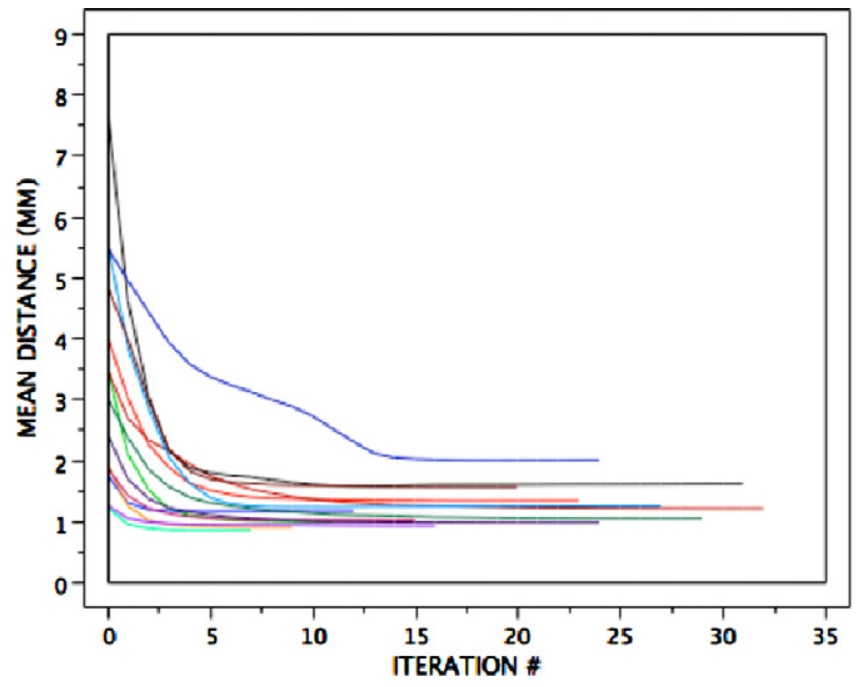

Fig. 3. Mean distance $(\mathrm{mm})$ between all source points and their closest target point as a function of iteration number for the 14 linear registrations presented in Tables 1 and 2. Iterations were stopped when the difference in mean distance between two successive iterations was smaller than $0.1 \mu \mathrm{m}$.

The results are presented in Table 1 and 2. These results show that the technique was capable of recovering on average $76 \%$ of the deformations ranging from 2 to $20 \mathrm{~mm}$ by measuring the distance between landmarks, with a maximum of $84 \%$ for a displacement of $-15 \mathrm{~mm}$ and a minimum of $53 \%$ for a displacement of $-6 \mathrm{~mm}$. By estimating the RMS over the ROI, we recovered $73 \%$ on average, with a maximum of $83 \%$ for a displacement of $-20 \mathrm{~mm}$ and a minimum of $50 \%$ for a displacement of $-6 \mathrm{~mm}$.

\subsection{Phantom study}

\subsubsection{Phantom preparation}

To further evaluate and validate the registration technique in a situation closer to a real clinical setting, we performed a phantom study. The phantom was made of

Table 1

Mean \pm std distance between 10 source and target landmarks before registration, after linear registration and after non-linear registration

\begin{tabular}{rrlll}
\hline $\begin{array}{l}\text { Displ. } \\
(\mathrm{mm})\end{array}$ & $\begin{array}{l}\text { Mean } \pm \text { std } \\
\text { before reg. }\end{array}$ & $\begin{array}{l}\text { Mean } \pm \text { std } \\
\text { after linear } \\
\text { reg. }\end{array}$ & $\begin{array}{l}\text { Mean } \pm \text { std } \\
\text { after non-linear } \\
\text { reg. }\end{array}$ & $\begin{array}{l}\% \text { of def. } \\
\text { recovered by } \\
\text { reg. }(\%)\end{array}$ \\
\hline-20 & $15.66 \pm 2.85$ & $2.83 \pm 1.57$ & $2.69 \pm 1.66$ & 83 \\
-15 & $11.63 \pm 2.27$ & $2.14 \pm 1.31$ & $1.88 \pm 1.06$ & 84 \\
-10 & $7.65 \pm 1.62$ & $1.63 \pm 1.10$ & $1.43 \pm 0.61$ & 81 \\
-8 & $6.08 \pm 1.33$ & $1.32 \pm 0.71$ & $1.19 \pm 0.54$ & 80 \\
-6 & $3.15 \pm 1.60$ & $1.53 \pm 1.13$ & $1.47 \pm 0.98$ & 53 \\
-4 & $3.00 \pm 0.71$ & $1.02 \pm 0.46$ & $0.86 \pm 0.34$ & 71 \\
-2 & $1.49 \pm 0.36$ & $0.54 \pm 0.32$ & $0.52 \pm 0.31$ & 65 \\
2 & $1.47 \pm 0.39$ & $0.52 \pm 0.10$ & $0.37 \pm 0.14$ & 75 \\
4 & $2.91 \pm 0.79$ & $0.81 \pm 0.43$ & $0.74 \pm 0.24$ & 75 \\
6 & $4.33 \pm 1.22$ & $1.30 \pm 0.65$ & $1.17 \pm 0.46$ & 73 \\
8 & $5.73 \pm 1.66$ & $1.55 \pm 0.99$ & $1.05 \pm 0.38$ & 82 \\
10 & $7.10 \pm 2.13$ & $2.08 \pm 1.22$ & $1.56 \pm 0.52$ & 78 \\
15 & $10.41 \pm 3.41$ & $2.88 \pm 1.66$ & $2.11 \pm 0.85$ & 80 \\
20 & $13.54 \pm 4.83$ & $3.64 \pm 1.59$ & $2.86 \pm 1.24$ & 79 \\
\hline
\end{tabular}


Table 2

3D RMS before registration, after linear registration and after non-linear registration evaluated over the region of interest (ROI)

\begin{tabular}{rllll}
\hline $\begin{array}{l}\text { Displ. } \\
(\mathrm{mm})\end{array}$ & $\begin{array}{l}\text { RMS (ROI) } \\
\text { before reg. }\end{array}$ & $\begin{array}{l}\text { RMS(ROI) } \\
\text { after linear } \\
\text { reg. }\end{array}$ & $\begin{array}{l}\text { RMS(ROI) } \\
\text { after non-linear } \\
\text { reg. }\end{array}$ & $\begin{array}{l}\text { \% of def. } \\
\text { recovered by } \\
\text { reg. }(\%)\end{array}$ \\
\hline-20 & 14.81 & 2.63 & 2.46 & 83 \\
-15 & 10.98 & 2.14 & 1.96 & 82 \\
-10 & 7.21 & 1.66 & 1.59 & 78 \\
-8 & 5.73 & 1.29 & 1.27 & 78 \\
-6 & 3.09 & 1.63 & 1.55 & 50 \\
-4 & 2.83 & 0.96 & 0.92 & 67 \\
-2 & 1.40 & 0.54 & 0.54 & 62 \\
2 & 1.38 & 0.52 & 0.36 & 74 \\
4 & 2.74 & 0.84 & 0.71 & 74 \\
6 & 4.08 & 1.33 & 1.19 & 71 \\
8 & 5.40 & 1.65 & 1.29 & 76 \\
10 & 6.69 & 2.15 & 1.59 & 76 \\
15 & 9.82 & 3.07 & 2.18 & 78 \\
20 & 12.79 & 4.64 & 3.74 & 71 \\
\hline
\end{tabular}

All measurements in $\mathrm{mm}$.

polyvinyl(alcohol)-cryogel (PVAc), and was designed to resemble a hemisphere of the human brain. To prepare the PVAc, the technique proposed by Surry et al. (2004) was applied. An inflatable $5 \mathrm{ml}$ Bardex Foley catheter (C.R. Bard, Inc., Covington, GA) was placed under the phantom to simulate a brain lesion, and plastic tubes with inside diameters of $1.57,2.36$ and $3.18 \mathrm{~mm}$ were inserted to simulate blood vessels. By inflating or deflating the catheter balloon, the phantom would deform in an elastic non-linear manner. A detailed description of the phantom as well as a thorough study of the reproducibility of the deformations can be found in Reinertsen and Collins (2006). The phantom made it possible to test the registration algorithm and segmentation technique as well as the ultrasound imaging setup and the navigation software in a setting with known geometry and simpler deformations than in the human brain. Because both MR and ultrasound images of the phantom were obtained both in the original and two deformed states, it was possible to validate the US based registration by comparing it to MR based registration. In this experiment, the MR based registration would therefore serve as a gold standard in order to validate the ultrasound based registration. A photo of the phantom is shown in Fig. 4.

\subsubsection{MR imaging and vessel segmentation}

The phantom was scanned using a Siemens SonataVision $1.5 \mathrm{~T}$ scanner using a standard $\mathrm{T} 1$ weighted anatomical scanning sequence $(\mathrm{TR}=22 \mathrm{~ms}, \mathrm{TE}=9.2 \mathrm{~ms}$, flip angle $=30^{\circ}$ ) with full brain coverage and $1 \mathrm{~mm}$ isotropic resolution. The phantom was scanned six times: twice for each catheter balloon volume filling $(0 \mathrm{ml}, 5 \mathrm{ml}$ and $10 \mathrm{ml})$. The catheter was either inflated or deflated between each scan. The inflation or deflation of the balloon deformed the phantom in a non-linear fashion as shown in Fig. 5. During MR imaging the phantom remained in the plastic container and the plastic tubes were filled with water. For

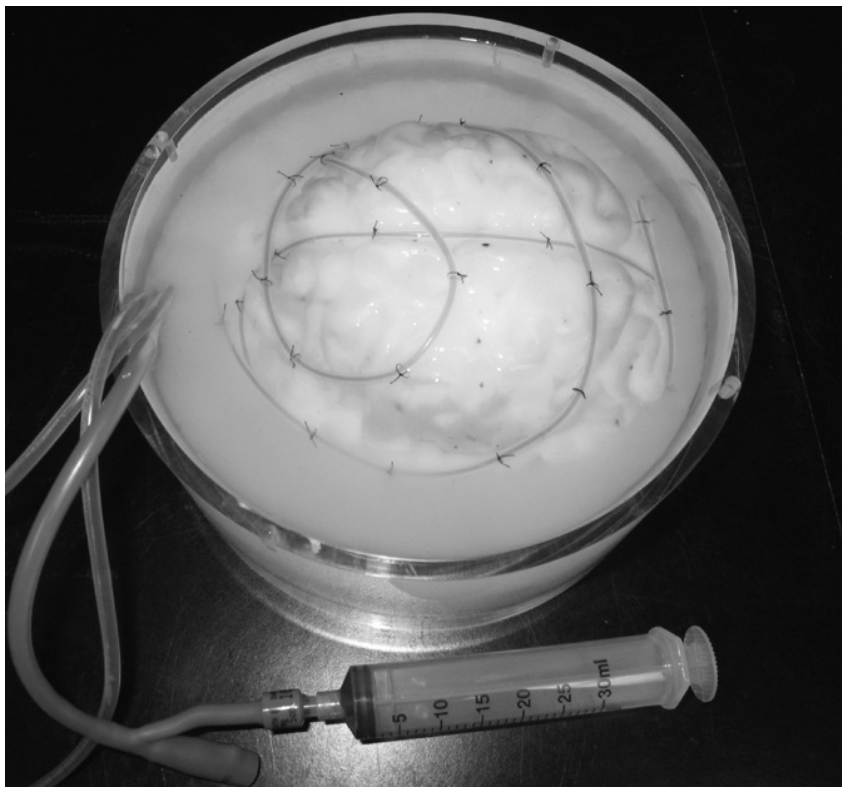

Fig. 4. The PVA phantom in a plastic container. The syringe was used to inject water into the catheter balloon to deform the phantom.

technical reasons there was no flow in the tubes during imaging, but due to the contrast between the PVA (bright), the tubes (dark) and the water inside the tubes (bright) it was still possible to apply the segmentation algorithm
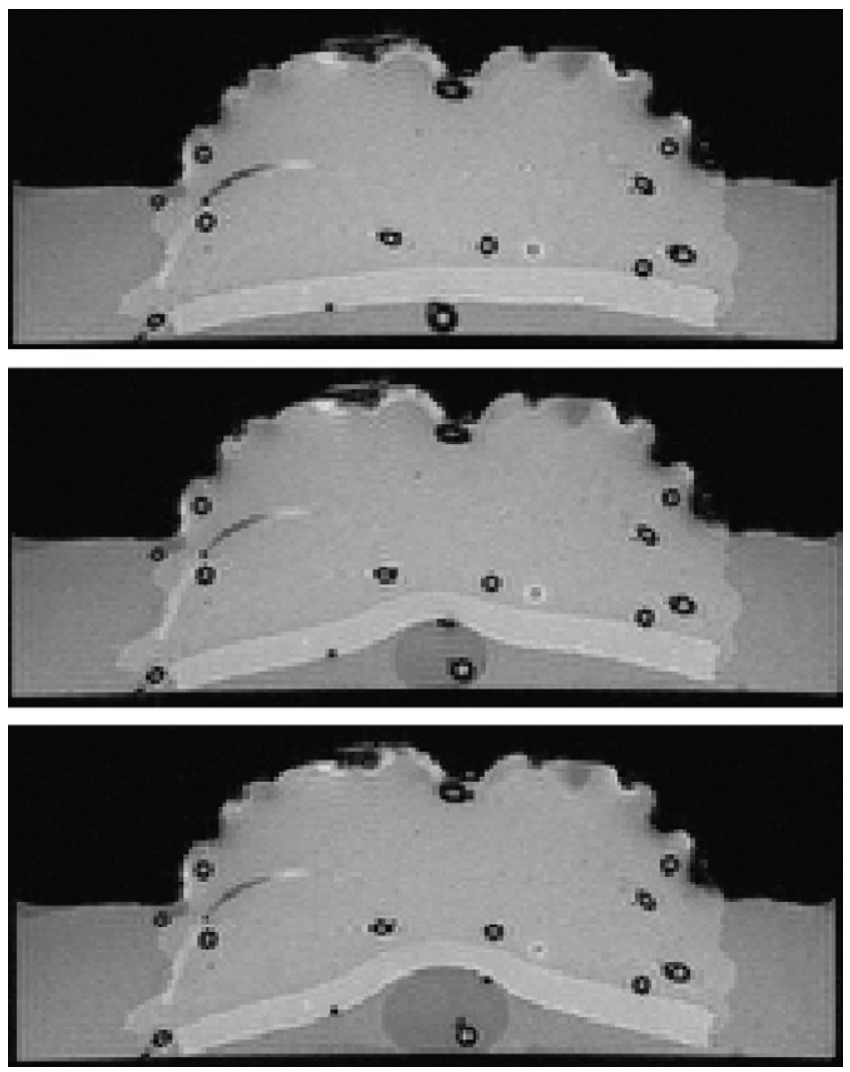

Fig. 5. MR images of the phantom with empty catheter balloon (top), half-full catheter balloon (middle) and full catheter balloon (bottom). 
described in Section 2.1. In order to be able to segment the smaller tubing, the original image was supersampled to $0.5 \mathrm{~mm}^{3}$ isotropic resolution. The smallest tubes used in the phantom have an inside diameter of $1.57 \mathrm{~mm}$. Unfortunately these tubes were too small for the automatic segmentation algorithm to detect due to limited contrast between the water inside the tubes and the plastic. For successful registration of the phantom data, the segmentation of all the tubes was necessary. This was mainly due to the limited number of tubes present in the phantom, and thus a limited number of tubes to capture the deformation. To overcome this problem, parts of the smallest tubes were segmented manually. In the future, a possible solution to this problem would be to fill the tubes with a contrast agent prior to MR imaging Reinertsen and Collins, 2006. Following segmentation, centerlines were extracted using the algorithm described in Section 2.3. A surface rendering of the phantom with the segmented tubes is shown in Fig. 6.

\subsubsection{US imaging and segmentation}

Free-hand ultrasound images were then acquired using an HDI 5000, Philips Medical Sytems (Bothwell, WA) ultrasound machine with a Philips P7-4 multi-frequency probe. Tracking was achieved with the Polaris optical tracking system (Northern Digital Inc., Waterloo, ON), a passive reference and an passive tracker device (Traxtal Inc., Toronto, ON) attached to the ultrasound probe. The position and orientation of each $2 \mathrm{D}$ image were recorded and used to reconstruct a $3 \mathrm{D}$ volume as described in Section 2.2. A physiological pump (Manostat Corp., New York City, NY) was used to pump water through the plastic tubes while the phantom was scanned using

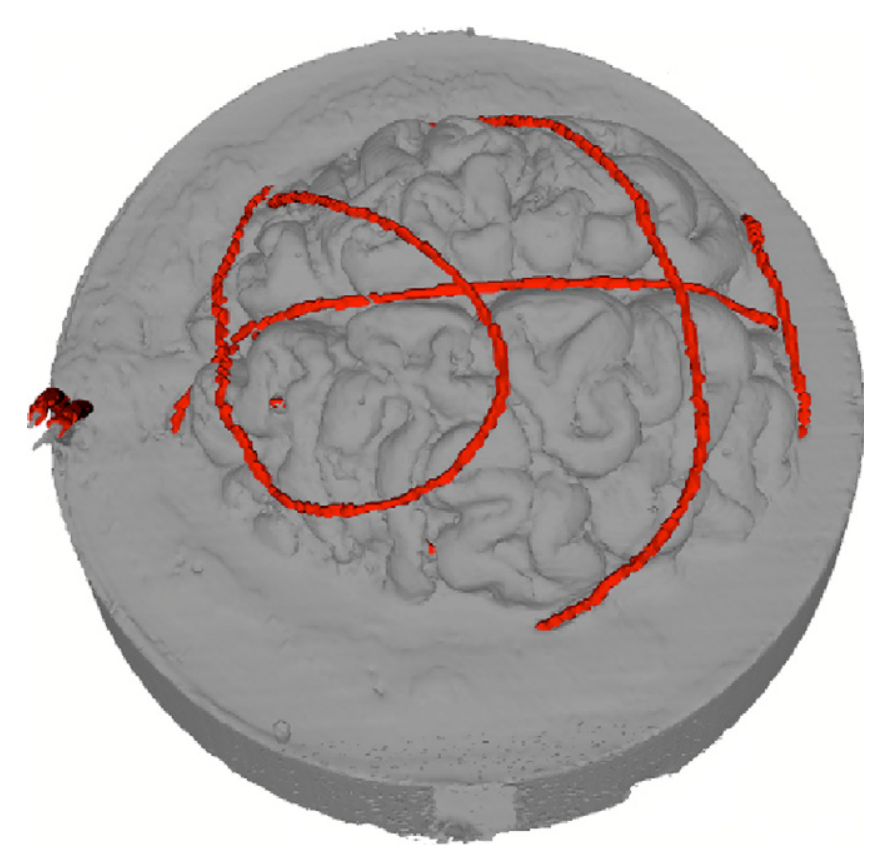

Fig. 6. A surface rendering of the phantom with the segmented tubes in red. (For interpretation of the references in color in this figure legend, the reader is referred to the web version of this article.) color Doppler imaging. The plastic container with the phantom was filled with water, and the phantom was allowed to rest for a few minutes for air bubbles in the water to disappear. This procedure is analogous to the one used in surgery when the craniotomy is filled with sterile water prior to ultrasound imaging. During Doppler imaging, the Doppler signal is overlaid on the regular Bmode ultrasound image. The gain of the B-mode signal was therefore turned down to facilitate the extraction of the "vessels" from the images afterwards. The phantom was scanned with catheter balloon filled with volumes of 0,5 and $10 \mathrm{ml}$ of water. The two dimensional ultrasound images were masked to remove all data outside the ultrasound image wedge, and then thresholded to separate the
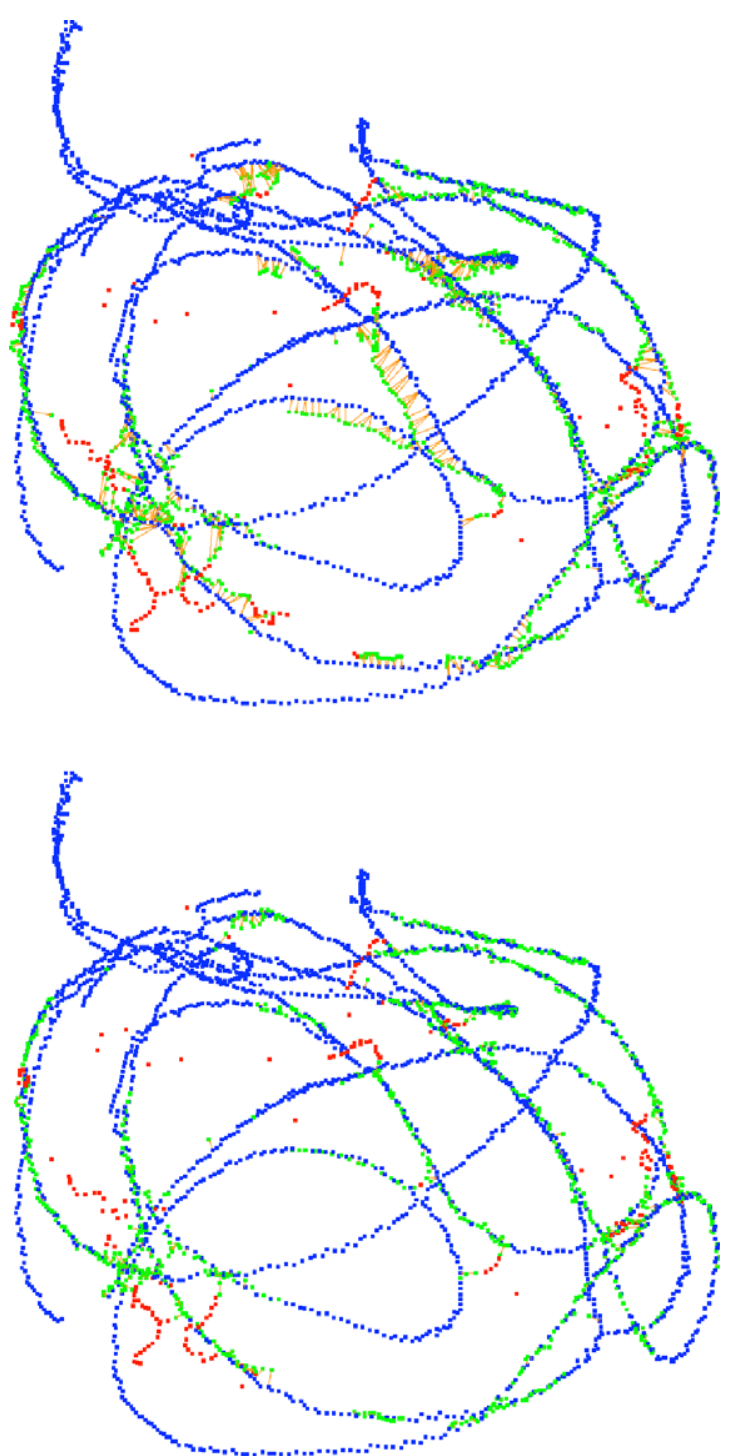

Fig. 7. US-MR registration: before (top) and after (bottom) non-linear registration. Target points are in blue, source points that participate in the registration are in green and source points that do not participate in the registration are in red. The yellow lines illustrate the closest point correspondences for the points that participate in the registration. (For interpretation of the references in color in this figure legend, the reader is referred to the web version of this article.) 
Doppler signal from the B-mode image. The slices were then resampled into a $3 \mathrm{D}$ volume. Following volume reconstruction, centerlines were extracted using the algorithm described in Section 2.3.

\subsubsection{Linear registration}

Prior to ultrasound imaging, the phantom was linearly registered to the MR images by identifying four homologous landmarks on the phantom container and in the corresponding MR image. In order to improve this initial alignment we performed a linear registration between the ultrasound and MR images with corresponding catheter balloon volumes as described in Section 2.4. In this case the ultrasound volume was considered the source volume and the MR volume the target because the ultrasound volume contained fewer vessels than the MR volume. Based on the results presented in Fig. 3, the iterations were stopped after 20 cycles. As we had six MR volumes and only three ultrasound volumes, each ultrasound volume was registered to both MR volumes with corresponding deformation, resulting in six linearly registered ultrasound volumes. These volumes provided the starting points for the non-linear registration described in the following section.

\subsubsection{Non-linear registration}

The centerlines of the MRI data and the US data were registered using the technique described in Section 2.4. Between $55 \%$ and $99 \%$ of the available points were used for registration. We used a scaling parameter $\sigma$ between 0.7 and 1.2. To decrease the computation time, the data were sub-sampled by a ratio between 0.5 (every second point) to 0.25 (every fourth point). The percentage of source points used, the sample ratio and $\sigma$ were manually optimized for each registration depending on the amount of noise, missing vessels and volume covered by the ultrasound. One example of the original centerlines extracted from the two volumes and the centerlines with the selected points and the initial pairings is shown in Fig. 7. We performed non-linear registrations between all the linearly registered ultrasound volumes to all MR volumes resulting in a total of 24 registrations.

In order to validate the accuracy of the registration we used a series of 10 homologous landmarks. Because it was very difficult to identify points in the ultrasound volume accurately, we tracked a series of points as they were deformed in the MR volumes. We identified 10 landmarks in all six MR volumes. These points were air bubbles in the PVA and less than $2 \mathrm{~mm}$ in diameter. They were clearly visible in all scans. The landmarks were located in the region of the phantom that deformed the most when the catheter balloon was either inflated or deflated. They were placed between "vessels" and did not participate in the registration. The transformation recovered after each non-linear registration was used to warp the landmarks identified in the source image, and the distances between the warped landmarks and the real landmarks identified in the target image were recorded. The mean distances of the landmarks in the source and target image before non-linear registration is shown in Table 3, and the distances between the warped landmarks and the landmarks in the target image are shown in Table 4. For comparison and in order to establish a lower bound on the registration error, we repeated the non-linear registrations using only MR data. One example of the MR-to-MR registration is shown in Fig. 8. In this case, we had full volume coverage for both source and target datasets and the overlap of the segmented vessels was nearly complete.

The mean distances between the warped landmarks and the real landmarks identified in the target image are shown

Table 3

Mean \pm std distance in $\mathrm{mm}$ between the 10 landmarks before non-linear registration

\begin{tabular}{lllllll}
\hline & $0 \mathrm{ml}-1$ & $0 \mathrm{ml}-2$ & $5 \mathrm{ml}-1$ & $5 \mathrm{ml}-2$ & $10 \mathrm{ml}-1$ \\
\hline $0 \mathrm{ml}-1$ & $\times$ & $\times$ & $1.64 \pm 0.56$ & $1.79 \pm 0.78$ & $3.69 \pm 0.94$ & $3.83 \pm 1.00$ \\
$0 \mathrm{ml}-2$ & $\times$ & $\times$ & $1.64 \pm 0.73$ & $1.78 \pm 0.77$ & $3.69 \pm 1.02$ \\
$5 \mathrm{ml}-1$ & $1.64 \pm 0.56$ & $1.64 \pm 0.73$ & $\times$ & $\times$ & $2.13 \pm 0.69$ & $3.83 \pm 1.07$ \\
$5 \mathrm{ml}-2$ & $1.79 \pm 0.78$ & $1.78 \pm 0.77$ & $\times$ & $\times$ & $2.32 \pm 0.64$ \\
$10 \mathrm{ml}-1$ & $3.69 \pm 0.94$ & $3.69 \pm 1.02$ & $2.14 \pm 0.69$ & $2.02 \pm 0.40$ & $\times 2 \pm 0.40$ \\
$10 \mathrm{ml}-2$ & $3.83 \pm 1.00$ & $3.83 \pm 1.07$ & $2.33 \pm 0.65$ & $2.21 \pm 0.44$ & $\times$ & $2.21 \pm 0.44$ \\
\hline
\end{tabular}

Table 4

Mean \pm std distance in $\mathrm{mm}$ between the 10 landmarks after US-to-MR non-linear registration

\begin{tabular}{lllllll}
\hline & $0 \mathrm{ml}-1$ & $0 \mathrm{ml}-2$ & $5 \mathrm{ml}-1$ & $5 \mathrm{ml}-2$ & $10 \mathrm{ml}-1$ \\
\hline $0 \mathrm{ml}-1$ & $\times$ & $\times$ & $1.07 \pm 0.53$ & $1.46 \pm 0.65$ & $1.50 \pm 0.78$ & $10 \mathrm{ml}-2$ \\
$0 \mathrm{ml}-2$ & $\times$ & $\times$ & $1.26 \pm 0.51$ & $1.23 \pm 0.68$ & $1.70 \pm 0.98$ \\
$5 \mathrm{ml}-1$ & $0.92 \pm 0.47$ & $0.91 \pm 0.39$ & $\times$ & $\times$ & $1.39 \pm 0.36$ & $1.85 \pm 1.23$ \\
$5 \mathrm{ml}-2$ & $1.32 \pm 0.62$ & $0.90 \pm 0.50$ & $\times$ & $1.92 \pm 0.56$ \\
$10 \mathrm{ml}-1$ & $2.11 \pm 0.92$ & $2.08 \pm 0.80$ & $1.98 \pm 1.02$ & $1.38 \pm 0.45$ & $\times$ & $1.51 \pm 0.38$ \\
$10 \mathrm{ml}-2$ & $2.69 \pm 0.76$ & $2.49 \pm 0.89$ & $1.74 \pm 0.57$ & $1.61 \pm 0.50$ & $\times$ & $\times$ \\
\hline
\end{tabular}

Ultrasound volumes (source) are listed vertically and MR volumes (target) horizontally. 

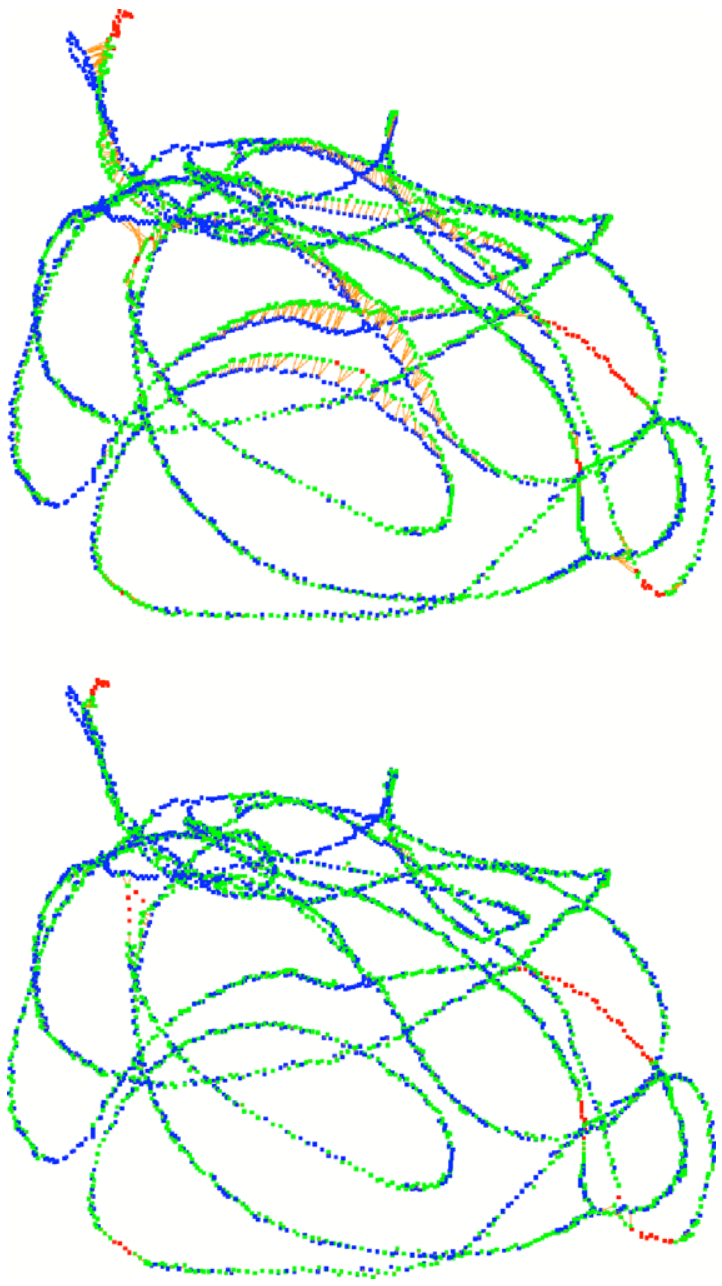

Fig. 8. MR-MR registration: before (top) and after (bottom) non-linear registration. Target points are in blue, source points that participate in the registration are in green and source points that do not participate in the registration are in red. The yellow lines illustrate the closest point correspondences for the points that participate in the registration. (For interpretation of the references in color in this figure legend, the reader is referred to the web version of this article.)

in Table 5 for the MR-MR experiment. Overall, these results show that we were able to correct the non-linear deformations with an average residual error of $1.6 \mathrm{~mm}$ for the ultrasound based registration. For comparison, the technique corrected the same deformations with an average residual error of $1.07 \mathrm{~mm}$ for the MR-to-MR registration.

\section{Discussion}

In this paper, we have presented a new method for correction of brain shift based on blood vessel segmentation and registration. The technique has been tested in a series of simulation experiments, and in a phantom study. It has shown to be able to recover large portions of linear and non-linear deformations even when only a very limited region of the MR image is covered by the US acquisition.

For the simulation experiments presented here, the technique was capable of recovering on average $75 \%$ of the deformations within the ROI with only $2 \%$ of the brain volume used to estimate the transformation. Because the ground truth was known in these experiments, there was no observer error associated with the identification of landmarks. Three of the registrations showed no improvement with non-linear registration. By visual inspection of the registration results, the alignment of the vessel trees improved, but this change was too small to influence the RMS or the landmarks.

The registrations presented in this paper can all be performed in less than $30 \mathrm{~s}$ on a $1.7 \mathrm{GHz}$ PC. Linear and nonlinear registration of the vessels can therefore be achieved in less than a minute. Non-linear resampling of entire image volumes might take more time. The computation speed is an important feature for intraoperative use, and will make it possible to efficiently correct preoperative data several times during a neurosurgical procedure. For the segmentation techniques, the most time consuming MR vessel segmentation can be computed pre-operatively and therefore does not add to the computation time required during surgery. The ultrasound vessel segmentation is only a simple thresholding, and the center line extraction can also be performed within less than $30 \mathrm{~s}$ which makes it possible to produce corrected anatomical and angiographic MR images in 1-2 min.

For the phantom study, it proved to be difficult to obtain a reliable segmentation of the smallest tubes from the MR images, especially the tubes on the surface of the phantom. This could probably be solved with higher resolution image acquisition, and if necessary a MR contrast agent in the tubes instead of water. However, this problem will not arise in real data sets were the blood vessels appear bright on a dark background (MRA, CTA) or dark on a bright background (PD) with no contrast between the vessel wall and the surrounding brain tissue.

Table 5

Mean \pm std distance in $\mathrm{mm}$ between the 10 landmarks after MR-to-MR non-linear registration

\begin{tabular}{|c|c|c|c|c|c|c|}
\hline & $0 \mathrm{ml}-1$ & $0 \mathrm{ml}-2$ & $5 \mathrm{ml}-1$ & $5 \mathrm{ml}-2$ & $10 \mathrm{ml}-1$ & $10 \mathrm{ml}-2$ \\
\hline $0 \mathrm{ml}-1$ & $x$ & $\times$ & $0.63 \pm 0.36$ & $0.87 \pm 0.47$ & $1.08 \pm 0.63$ & $1.35 \pm 0.54$ \\
\hline $0 \mathrm{ml}-2$ & $\times$ & $\times$ & $0.77 \pm 0.51$ & $0.73 \pm 0.36$ & $1.17 \pm 0.64$ & $1.50 \pm 0.62$ \\
\hline $5 \mathrm{ml}-1$ & $0.73 \pm 0.42$ & $0.75 \pm 0.25$ & $\times$ & $\times$ & $0.94 \pm 0.43$ & $0.95 \pm 0.45$ \\
\hline $5 \mathrm{ml}-2$ & $0.90 \pm 0.40$ & $0.82 \pm 0.55$ & $\times$ & $\times$ & $0.87 \pm 0.56$ & $1.17 \pm 0.47$ \\
\hline $10 \mathrm{ml}-1$ & $1.33 \pm 0.82$ & $1.43 \pm 0.96$ & $1.19 \pm 0.54$ & $1.00 \pm 0.34$ & $\times$ & $\times$ \\
\hline $10 \mathrm{ml}-2$ & $1.63 \pm 0.89$ & $1.59 \pm 0.86$ & $1.04 \pm 0.58$ & $1.21 \pm 0.49$ & $x$ & $x$ \\
\hline
\end{tabular}


Missing ultrasound data in highly deformed regions, in particular the tubes on the top of the phantom surface limited the accuracy of the registration. The ultrasound acquisition therefore has to be optimized for registration purposes, in order to target vessels in highly deformed regions. Despite these difficulties, the US-to-MR registration was able to recover the deformations to within an average of $1.6 \mathrm{~mm}$ compared to an average of $1.07 \mathrm{~mm}$ for the MR-to-MR registration. We consider the MR-toMR registration the best possible result for this technique, and it shows an accuracy comparable to the resolution of the original data and the observer error in point identification.

These results demonstrate that ultrasound imaging in general and Doppler ultrasound in particular can be very useful modalities in detection and correction of brain shift occurring during neurosurgical operations if the ultrasound acquisition is carefully performed in the highly deformed regions and optimized in order to capture vessels that are well represented in the preoperative MRA data. Better segmentation techniques for MRA data that include segmentation of smaller vessels and in particular vessels on the cortical surface will increase the accuracy of the image registration.

Even though numerical simulations and physical phantoms are useful to test validate a registration technique, these approximations cannot fully simulate the complexity of the human brain. The registration technique will therefore be applied to a series of clinical data in the near future. The application of the technique to real data will enable us test the technique on data with anatomical variability, different magnitudes and directions of brain shift, and different ultrasound volume coverage. This application will also enable us to make further improvements to the navigation software and the registration algorithm. Classification of vessel segments and branching points in order to further reduce the number of incorrect pairings and take into account vessel directions are possible improvements to the existing algorithm.

\section{Conclusions}

In this study, we have designed and validated a method to detect and correct brain shift using image registration of blood vessels segmented from MR images and Doppler ultrasound data. The registration algorithm can correct the deformed region to within 1-2 mm. The ultrasound based registration was compared to results obtained using MR-to-MR registration, and the results are comparable taking into account the difference in volume coverage. While more experiments are required to test the method with real patient data, these experiments show that blood vessels have the potential of being very useful features for registration of MR and US images. By using segmented blood vessels, we overcome many of the difficulties associated with registration of US data, providing the neurosurgeon with a fast tool to obtain accurate information about the anatomy and vasculature at any point in time during a surgical procedure.

\section{Acknowledgements}

We are grateful to Sylvain Bouix for providing his code for the centerline extraction algorithm.

\section{References}

Arbel, T., Morandi, X., Comeau, R.M., Collins, D.L., 2001. Automatic non-linear MRI-ultrasound registration for the correction of intraoperative brain deformations. In: Proc. MICCAI 2001, pp. 913-922.

Arbel, T., Morandi, X., Comeau, R.M., Collins, D.L., 2001. Automatic non-linear mri-ultrasound registration for the correction of intraoperative brain deformation. Computer Aided Surgery 9 (4), 123-136.

Aylward, S.R., Jomier, J., Jean-Philippe Guyon, Weeks, S., 2002. Intraoperative 3D ultrasound augmentation. In: Proc. IEEE International Symposium on Biomedical Imaging, pp. 421-424.

Aylward, S.R., Jomier, J., Weeks, S., Bullitt, E., 2003. Registration and analysis of vascular images. International Journal of Computer Vision $55(2 / 3), 123-138$

Besl, P.J., McKay, N.D., 1992. A method for registration of 3D shapes. IEEE PAMI 14 (2), 239-256.

Black, P.M., Alexander, E., Martin, C., Moriarty, T., Nabavi, A., Wong, T.Z., Schwartz, R.B., Jolesz, F., 1999. Craniotomy for tumor treatment in an intraoperative magnetic resonance imaging unit. Neurosurgery 45 (3), 423-433.

Bookstein, F.L., 1989. Principal warps: thin-plate splines and the decomposition of deformations. IEEE PAMI 11 (6), 567-585.

Bouix, S., Siddiqi, K., Tannenbaum, A., 2005. Flux driven automatic centerline extraction. Medical Image Analysis 9, 209-221.

Chen, Y., Medioni, G., 1991. Object modeling by registration of multiple range images. IEEE Conference on Robotics and Automation.

Chetverikov, D., Svirko, D., Stepanov, D., 2002. The trimmed iterative closest point algorithm. In: Proc. International Conference on Pattern Recognition.

Collins, D.L., Evans, A.C., 1997. ANIMAL: Validation and application of non-linear registration-based segmentation. IJPRAI 11 (8), 12711294.

Comeau, R.M., Sadikot, A.F., Fenster, A., Peters, T.M., 2000. Intraoperative ultrasound for guidance and tissue shift correction in imageguided neurosurgery. Medical Physics 27 (4), 787-800.

Descoteaux, M., 2004. A Multi-Scale Geometric Flow for Segmenting Vasculature in MRI: Theory and Validation, Master's Thesis, School of Computer Science, McGill University.

Ferrant, M., Nabavi, A., Macq, B., Black, P.M., Jolesz, F.A., Kikinis, R., Warfield, S.K., 2002. Serial registration of intraoperative MR images of the brain. Medical Image Analysis 6, 337-359.

Fischler, M.A., Bolles, R.C., 1981. Random sample consensus: a paradigm for model fitting with applications to image analysis and automated cartography. Communication of the ACM 24, 381-395.

Frangi, A.F., Niessen, W.J., Vincken, K.L., Viergever, M.A., 1998. Muliscale vessel enhancement filtering. In: Proc. MICCAI 1998, pp. $130-137$.

Gobbi, D.G., Comeau, R.M., Peters, T.M., 2000. Ultrasound/MRI overlay with image warping for neurosurgery. In: Proc. MICCAI 2000, pp. $106-114$

Grønningsæter, Aa., Kleven, A., Ommedal, S., Aarseth, T.E., Lie, T., Lindseth, F., Langø, T., Unsgaard, G., 2000. SonoWand, an ultrasound-based neuronavigation system. Neurosurgery 47 (6), 1373-1380.

Grunert, P., Muller-Forell, W., Darabi, K., Reisch, R., Busert, C., Hopf, N., Perneczky, A., 1998. Basic principles and clinical applications of neuronavigation and intraoperative computed tomography. Computer Aided Surgery 3 (4), 166-173. 
Haberland, N., Ebmeier, K., Hliscs, R., Grunewald, J.P., Silbermann, J., Steenbeck, J., Nowak, H., Kalff, R., 2000. Neuronavigation in surgery of intracranial and spinal tumors. Journal of Cancer Research and Clinical Oncology 126, 529-541.

Hartkens, T., Hill, D.L.G., Castellano-Smith, A.D., Hawkes, D.J., Maurer, C.R., Martin, A.J., Hall, W.A., Liu, H., Truwit, C.L., 2003. Measurement and analysis of brain deformation during neurosurgery. IEEE Transactions on Medical Imaging 22 (1), 82-92.

Hastreiter, P., Rezk-Salama, C., Soza, G., Bauer, M., Greiner, G., Fahlbusch, R., Ganslandt, O., Nimsky, C., 2004. Strategies for brain shift evaluation. Medical Image Analysis 8, 447-464.

Hill, D.L.G., Maurer, C.R., Maciunas, R.J., Barwise, J.A., Fitzpatrick, J.M., Wang, M.Y., 1998. Measurement of intraoperative brain surface deformation under a craniotomy. Neurosurgery 43 (3), 514-528.

Jolesz, F.A., 2005. Future perspectives for intraoperative MRI. Neurosurgery Clinics of North America 16 (1), 201-213.

Jomier, J., Aylward, S.R., 2004. Rigid and deformable vasculature-toimage registration: a hierarchical approach. In: Proc. MICCAI 2004, pp. 829-836.

Lange, T., Eulenstein, S., Hunerbein, M., Lamecker, H., Peter-Michael Schlag., 2004. Augmenting intraoperative 3D ultrasound with preoperative models for navigation in liver surgery. In: Proc. MICCAI 2004, pp. 534-541.

Letteboer, M.M., Willems, P.W.A., Viergever, M.A., Niessen, W.J., 2005. Brain shift estimation in image-guided neurosurgery using 3-D ultrasound. IEEE Transactions on Medical Imaging 52 (2).

Masuda, T., Sakaue, K., Yokoya, N., 1996. Registration and integration of multiple range images for 3-D model construction. Proceedings of ICPR.

Miga, M.I., Paulsen, K.D., Lemery, J.M., Eisner, S.D., Hartov, A., Kennedy, F.E., Roberts, D.W., 1999. Model-updated image gudence: initial clinical experiences with gravity-induced brain deformation. IEEE Transactions on Medical Imaging 18 (10), 866-874.

Nabavi, A., Black, P.M., Gering, D.T., Westin, C.F., Mehta, V., Pergolizzi Jr., R.S., Ferrant, M., Warfield, S.K., Hata, N., Schwartz, R.B., Wells 3rd., W.M., Kikinis, R., Jolesz, F.A., 2001. Serial intraoperative magnetic resonance imaging of brain shift. Neurosurgery 48 (4), 787-798.

Nimsky, C., Ganslandt, O., Hastreiter, P., Fahlbusch, R., 2001. Intraoperative compensation for brain shift. Surgical Neurology 10, $357-365$.

Nimsky, C., Ganslandt, O., von Keller, B., Romstock, J., Fahlbusch, R., 2004. Intraoperative high-field-strength MR imaging: implementation and experience in 200 patients. Radiology 233, 67-78.

Penney, G.P., Little, J.A., Weese, J., Hill, D.L.G., Hawkes, D.J., 2004. Registration of freehand 3D ultrasound and magnetic resonances liver images. Medical Image Analysis 8, 81-91.
Porter, B.C., Rubens, D.J., Strang, J.G., Smith, J., Totterman, S., Parker, K.J., 2001. Three-dimensional registration and fusion of ultrasound and mri using major vessels as fiducial markers. IEEE Transactions on Medical Imaging 20 (4), 354-359.

Pulli, K., 1999. Multiview registration for large data sets. In: Proc. 3DIM.

Reinertsen, I., Collins, D.L., 2006. A realistic phantom for brain-shift simulations. Medical Physics 33 (9), 3234-3240.

Reinertsen, I., Descoteaux, M., Drouin, S., Siddiqi, K., Collins, D.L., 2004. Vessel driven correction of brain shift. In: Proc. MICCAI 2004, pp. 208-216.

Roberts, D.W., Miga, M.I., Hartov, A., Eisner, S., Lemery, J.M., Kennedy, F.E., Paulsen, K.D., 1998. Intraoperative brain shift and deformation: a quantitative analysis of cortical displacement in 28 cases. Neurosurgery 43 (5), 749-760.

Roche, A., Pennec, X., Malandin, G., Ayache, N., 2001. Rigid registration of 3D ultrasound with mr images: a new approach combining intensity and gradient information. IEEE Transactions on Medical Imaging 20 (10), 1038-1049.

Rousseeuw, P.J., Leroy, A.M., 1987. Robust Regression and Outlier Detection, Wiley Series. In: Probability and Mathematical Statistics, first ed., 1987.

Rusinkiewicz, S., Levoy, M., 2001. Efficient Variants of the ICP Algorithm. In: Proc. 3rd International Conference on 3D Digital Imaging and Modeling.

Siddiqi, K., Bouix, S., Tannenbaum, A., Zucker, S.W., 2002. HamiltonJacobi skeletons. International Journal of Computer Vision 48 (3).

Skrinjar, O., Nabavi, A., Duncan, J., 2002. Model-driven brain shift compensation. Medical Image Analysis 6, 361-373.

Slomka, P.J., Mandel, J., Downey, D., Fenster, A., 2001. Evaluation of voxel-based registration of 3-D power Doppler ultrasound and 3-D magnetic resonance angiographic images of carotid arteries. Ultrasound in Medicine and Biology 27 (7), 945-955.

Soza, G., Nimsky, C., Greiner, G., Hastreiter, P., 2004. Estimating mechanical brain tissue properties with simulation and registration. In: Proc. MICCAI 2004, pp. 276-283.

Surry, K.J.M., Austin, H.J.B., Fenster, A., Peters, T.M., 2004. Poly(vinyl alcohol) cryogel phantoms for use in ultrasound and MR imaging. Physics in Medicine and Biology 49 (24), 5529-5546.

Trucco, E., Fusiello, A., Roberto, V., 1999. Robust motion and correspondence of noisy 3-D point sets with missing data. Pattern Recognition Letters 20, 889-898.

Turk, G., Levoy, M., 1994. Zippered Polygon Meshes from Range Images. In: Proc. SIGGRAPH.

Vasilevskiy, A., Siddiqi, K., 2002. Flux maximizing geometric flows. IEEE PAMI 24 (12), 1565-1578.

Weik, S., 1997. Registration of 3-D partial surface models using luminance and depth information. In: Proc. 3DIM. 